\title{
SCDI1:
}

\section{uma Ferramenta Computacional Educacional para a Visualização, Modelagem e Inversão de Funções Matemáticas}

\author{
Bruno Nunes Myrrha Ribeiro \\ Centro Universitário Geraldo Di Biase - UGB, Barra do Piraí-RJ \\ myrrhaugb@gmail.com
}

\begin{abstract}
Resumo: Este artigo apresenta o sistema computacional educacional SCDI 1 - Sistema de Cálculo Diferencial e Integral 1. Sua proposta pedagógica está direcionada a complementar o processo ensino-aprendizagem na disciplina de Cálculo I (Cálculo Diferencial e Integral), disciplina básica dos cursos de engenharia. Em sua primeira versão aborda tópicos elementares de funções matemáticas, possibilitando também, sua aplicação em conteúdos relativos às disciplinas de Matemática do ensino médio. Em sua primeira versão, seu objetivo principal é visualizar e modelar diversas funções em uma variável e suas inversas, em suas representações: algébrica, gráfica e numérica, permitindo a alteração dos coeficientes, área de visualização e bissetrizes. Como desenvolvimento e implementação futura, destacam-se assuntos relativos à combinação e composição, limites, continuidade, derivada e integral, de funções em uma variável.
\end{abstract}

Palavras-chave: Software educativo. Cálculo diferencial e integral. Funções matemáticas.

\section{SCDI1:}

\section{An Educational Tool for Computational Visualization, Modeling and Inversion of Mathematical Functions}

\begin{abstract}
This article presents the educational computer system SCDI 1 System of Differential and Integral Calculus 1. Its pedagogical proposal is aimed to complement the teaching-learning process in the course of Calculus I (Differential and Integral Calculus), basic discipline in engineering courses. In its first version, discusses topics of elementary mathematical functions, also allowing its application to contents related to the disciplines of mathematics high school. In its first version, the main goal is to visualize and model a variety
\end{abstract}


of functions in a variable and the inverse in its representations: algebraic, graphical and numerical; allowing the change of coefficients, the viewing area and bisectors. As a project with future implementation, we highlight issues concerning the combination and composition, limits, continuity, derivative and integral of functions in one variable.

Keywords: Educational software. Differential and integral calculus. Math function.

\section{Introdução}

O constante desenvolvimento das Tecnologias de Informação e Comunicação(TIC) implica no avanço de diversas possibilidades pedagógicas, caracterizando o desenvolvimento científico em relação ao ensino e a pesquisa, favorecendo diretamente o processo ensinoaprendizagem. Assume-se, então, a nova área temática, a Informática na Educação. Derivada das novas práticas pedagógicas, a prática educativa computacional é direcionada ao desenvolvimento do software educacional e suas avaliações em sala de aula.

“(...) a visão cada vez mais consensual na comunidade da Informática Educativa é a de que o contextualize no processo ensino-aprendizagem, pode ser considerado educacional.” (Giraffa, 1999)

A motivação para o desenvolvimento da ferramenta educacional apresentada vem de uma dificuldade constatada em relação à aprendizagem na disciplina de Cálculo I (Cálculo Diferencial e Integral), disciplina básica das áreas de Ciências Exatas e Engenharias, devido à falta de absorção de conhecimentos relativos a funções matemáticas elementares, apresentados ao longo do ensino médio. O sistema proposto é do tipo CAI (Computer Assisted Instruction - Instrução Auxiliada por Computador) e se baseia no contexto educacional em uma perspectiva construcionista (Papert, 1994).

Classificado como Ferramenta Educacional, o SCDI 1 - Sistema de Cálculo Diferencial e Integral 1 tem a finalidade de complementar o processo pedagógico da disciplina de Cálculo I, possibilitando a interatividade em sua utilização, permitindo a realização do ciclo descrição-execução-reflexão-depuração segundo Almeida (2000). Propõe como objetivo principal, a visualização e modelagem de diversas funções matemáticas em uma variável, e suas respectivas funções inversas, em suas representações: algébrica, gráfica e numérica (tabela). A forma de coordenar essas representações é denominada epistemologia das representações múltiplas, de acordo com Borba (2001):

“(...) o importante não é privilegiar um tipo apenas de representação e, sim diferentes representações para uma mesma função: algébrica, gráfico e tabela."

(...) propõem a coordenação entre elas como um novo caminho para o conhecimento de funções, ou seja, uma epistemologia das representações múltiplas. 
(...) essa nova abordagem só ganha força com ambientes computacionais que geram gráficos vinculados a tabelas e expressões algébricas."

Além de fornecer e reforçar a prática dos conteúdos abordados em sala de aula, também permite analisar e realizar experimentos, assumindo as características básicas de Sistema de Exercícios e Práticas e Simulação (Valente, 1999). Como referência para o desenvolvimento e proposta, destaca-se o software INTNUM (Myrrha, 2011), uma ferramenta computacional que complementa o processo ensino-aprendizagem do conteúdo "Métodos de Integração Numérica", relativo à disciplina de Cálculo Numérico.

O presente artigo se estrutura da seguinte forma: seção 2 - quadro teórico, seção 3 desenvolvimento computacional e seção 4 - resultados.

\section{Quadro Teórico}

Função em uma variável e função inversa são considerados um dos conceitos importantes na matemática, tem como objetivo, a descrição do comportamento relacional entre dois elementos (relação binária), com aplicabilidade multidisciplinar em diversas áreas científicas.

Definição 1: Função Matemática

Dado dois conjuntos $A, B \neq \emptyset \mathrm{e} f$ uma aplicação de ${ }^{A} \mathrm{em}^{B}$. Define-se função $f(x)=y$ onde:

$$
f: A \rightarrow B \quad{ }_{x \rightarrow f(x)} \Leftrightarrow \forall x \in A, \exists y \in B \mid(x, y) \in f
$$

$\operatorname{com}^{x}$ o valor de entrada e ${ }^{y}$ o valor de saída.

\section{Definição 2: Função Inversa}

Dado dois conjuntos $A, B \neq \emptyset$ e $f^{-1}$ uma aplicação de ${ }^{B}$ em ${ }^{A}$, sendo $f$ uma função bijetora. Define-se função inversa $f^{-1}(y)=x, \operatorname{com} f(x)=y$, onde:

$$
f^{-1}: B \rightarrow A \quad{ }_{y \rightarrow f^{-1}(y)} \Leftrightarrow \forall y \in B, \exists x \in A \mid(y, x) \in f^{-1}
$$

com $^{y}$ o valor de entrada $\mathrm{e}^{x}$ o valor de saída.

As representações são dadas por meio de expressões matemáticas, gráficos e tabela numérica. 


\section{Representação Algébrica}

$$
f\left(x_{i}\right)=a\left(b x_{i}+c\right)^{d}+e
$$

com a, b, c, d, e, os coeficientes da função.

\section{Representação Gráfica}

Representada por meio de curvas ou retas no plano cartesiano (Figura 1), através da correspondência entre os pares ordenados $(x, f(x))$.

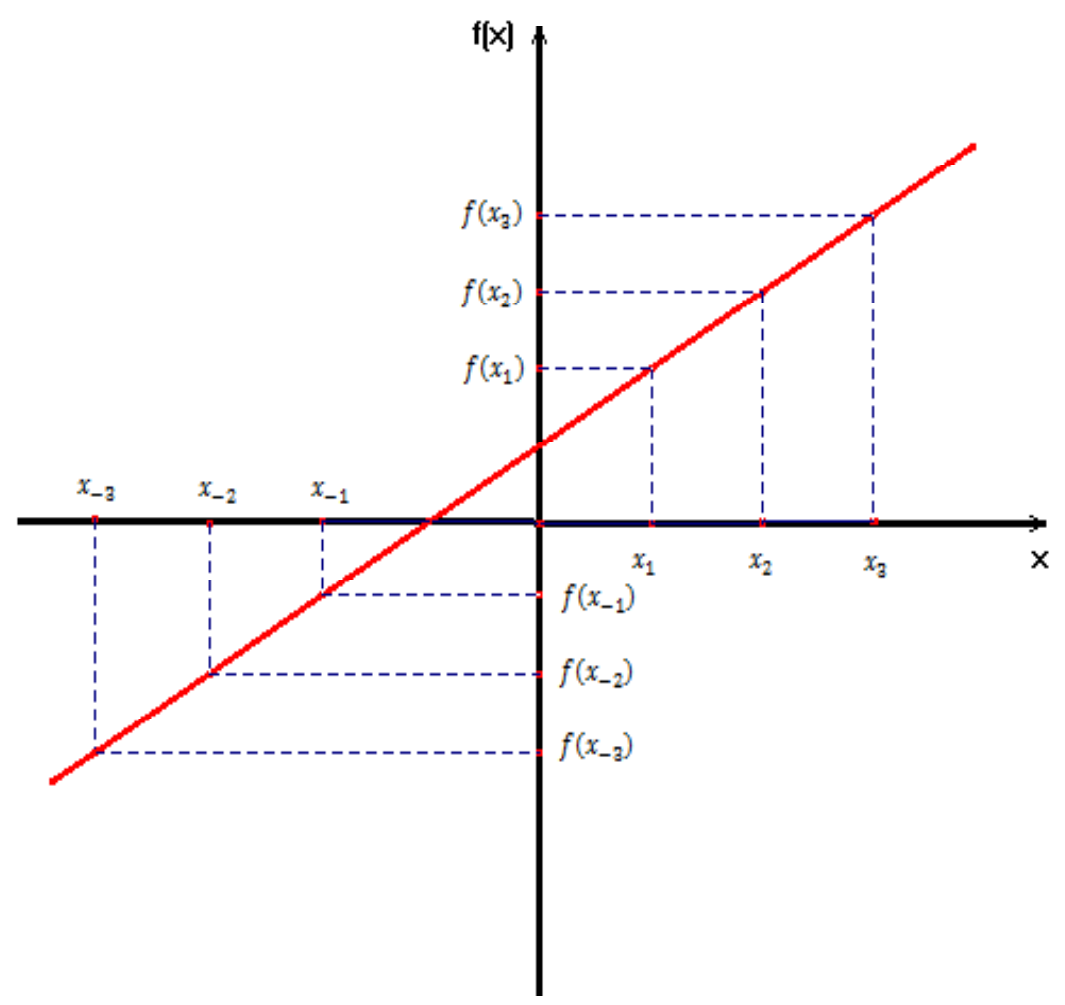

Figura 1. Representação Gráfica

\section{Representação Numérica}

A representação numérica é dada por meio de tabela de pares ordenados, correspondentes a pontos na função (Quadro 1 ), como mostra a tabela abaixo $(n \in \mathbb{R})$ : 
Quadro 1. Representação Numérica

\begin{tabular}{|c|c|}
\hline$x_{-n}$ & $f\left(x_{-n}\right)$ \\
\hline$x_{-n+1}$ & $f\left(x_{-n+1}\right)$ \\
\hline$\vdots$ & $\vdots$ \\
\hline$x_{0}$ & $f\left(x_{0}\right)$ \\
\hline$\vdots$ & $\vdots$ \\
\hline$x_{n-1}$ & $f\left(x_{n-1}\right)$ \\
\hline$x_{n}$ & $f\left(x_{n}\right)$ \\
\hline
\end{tabular}

\section{Desenvolvimento Computacional}

O software foi desenvolvido no ambiente Wolfram Mathematica 8 (http://www.wolfram. com) aplicado em sistema gráfico e de inteface.

\section{Wolfram Mathematica 8}

Software de computação algébrica com diversos recursos e linguagem própria, rico em procedimentos nos desenvolvimentos: simbólico, gráfico e numérico. Sua aplicabilidade engloba diversas áreas científicas, atuando fortemente no desenvolvimento de projetos na tríade Ensino, Pesquisa e Indústria, em diversos países.

"O software desempenhou um papel crucial em várias descobertas e tem servido de base para milhares de artigos científicos." (Wolfram, 2011)

Tornando-se, nas engenharias, algo indispensável.

"Na engenharia, o Mathematica se tornou o padrão tanto para pesquisa e desenvolvimento quanto para produção (...).” (Wolfram, 2011)

Considera-se como uma poderosa ferramenta em relação manipulação de dados, integrado em sistema de desenvolvimento de interface. 
“(...) também surgiu como uma ferramenta importante na ciência da computação e desenvolvimento de softwares: a linguagem empregada pelo Mathematica é utilizada amplamente em ambientes de desenvolvimento de pesquisa, protótipos e interfaces."(Wolfram, 2011)

Importantes fatores que contribuíram na escolha da ferramenta para o desenvolvimento do software SCDI 1.

\section{Resultado}

Apresenta-se o software na Figura 2, em seus parâmetros default com as possibilidades: algébrica, gráfica e numérica. Em sua interface, as interações são determinadas pelos "boxes" apresentados:

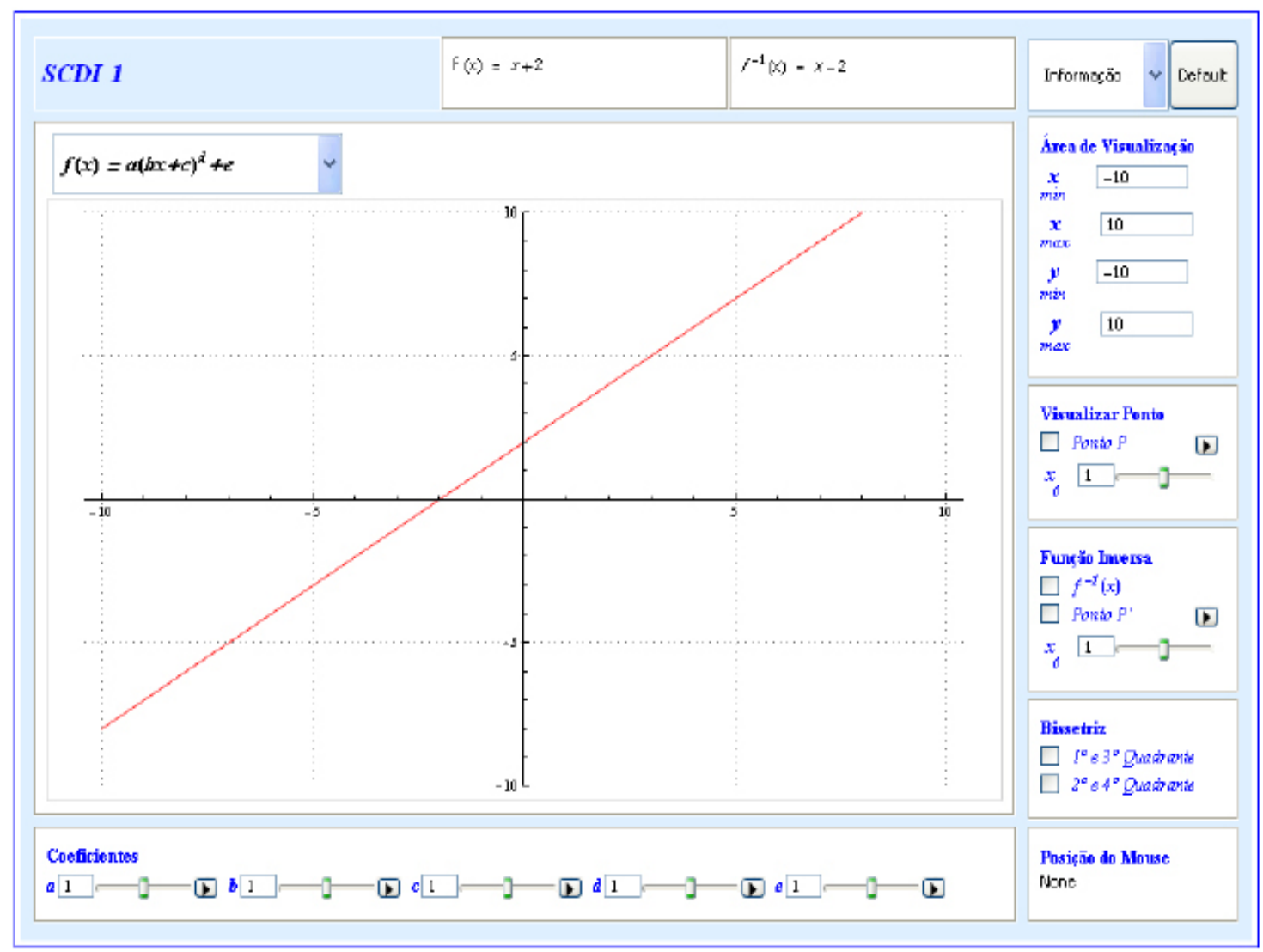

Figura 2. SCDI 1 (default), $f(x)=x+2$

\section{Seleção}

Permite a escolha das funções pré-determinadas, possibilitando sua modelagem, a partir da alteração de seus coeficientes, em suas representações gráficas, algébricas e numéricas.

As funções disponibilizadas no sistema são apresentadas em sua forma algébrica, no Quadro 2. 
Quadro 2. Funções Cadastradas

$$
\begin{aligned}
& f_{1}(x)=a(b x+c)^{d}+e \\
& f_{2}(x)=\left\{\begin{array}{l}
(a x+b)^{d}, \text { se } x \geq e \\
(a x+d)^{b}, \text { se } x<e
\end{array}\right. \\
& f_{3}(x)=a^{(b x+c)^{d}}+e \\
& f_{4}(x)=\log _{a}(b x+c)^{d}+e \\
& f_{5}(x)=a \operatorname{Sen}(b x+c)^{d}+e \\
& f_{6}(x)=a \operatorname{Cos}(b x+c)^{d}+e \\
& f_{7}(x)=a \operatorname{Tan}(b x+c)^{d}+e \\
& f_{8}(x)=a \operatorname{Cot}(b x+c)^{d}+e \\
& f_{9}(x)=a \operatorname{Sec}(b x+c)^{d}+e \\
& f_{10}(x)=a \operatorname{Csc}(b x+c)^{d}+e
\end{aligned}
$$

\section{Área de Visualização}

Alteração na visualização dos eixos coordenados (x - abscissa e y - ordenada). Com $x_{\min }, x_{\max }, y_{\min }$ e $y_{\max }$, os dados de entrada.

\section{Visualizar Ponto}

Visualização de um ponto $P\left(x_{0}, y_{0}\right)$ na função e suas coordenadas, com $x_{0}$ o parâmetro de entrada, possibilita uma interatividade através da alteração dinâmica com o slider (barra horizontal). Outra possibilidade é a animação, através de um player, permitindo a visualização de ponto percorrendo toda a função (Figura 3). Objetivando na representação numérica da função em uma variável.

\section{Função Inversa}

Visualização da função inversa $f^{-1}(x)$ e do ponto $P^{\prime}\left(x_{0}, y_{0}\right)$ pertencente a inversa. Permite fatores de interação e animação (slider e player), como se mostra na Figura 3. 
SCDI 1: Uma Ferramenta Computacional Educacional para a Visualização, Modelagem e Inversão de Funções Matemáticas

Bruno Nunes Myrrha Ribeiro

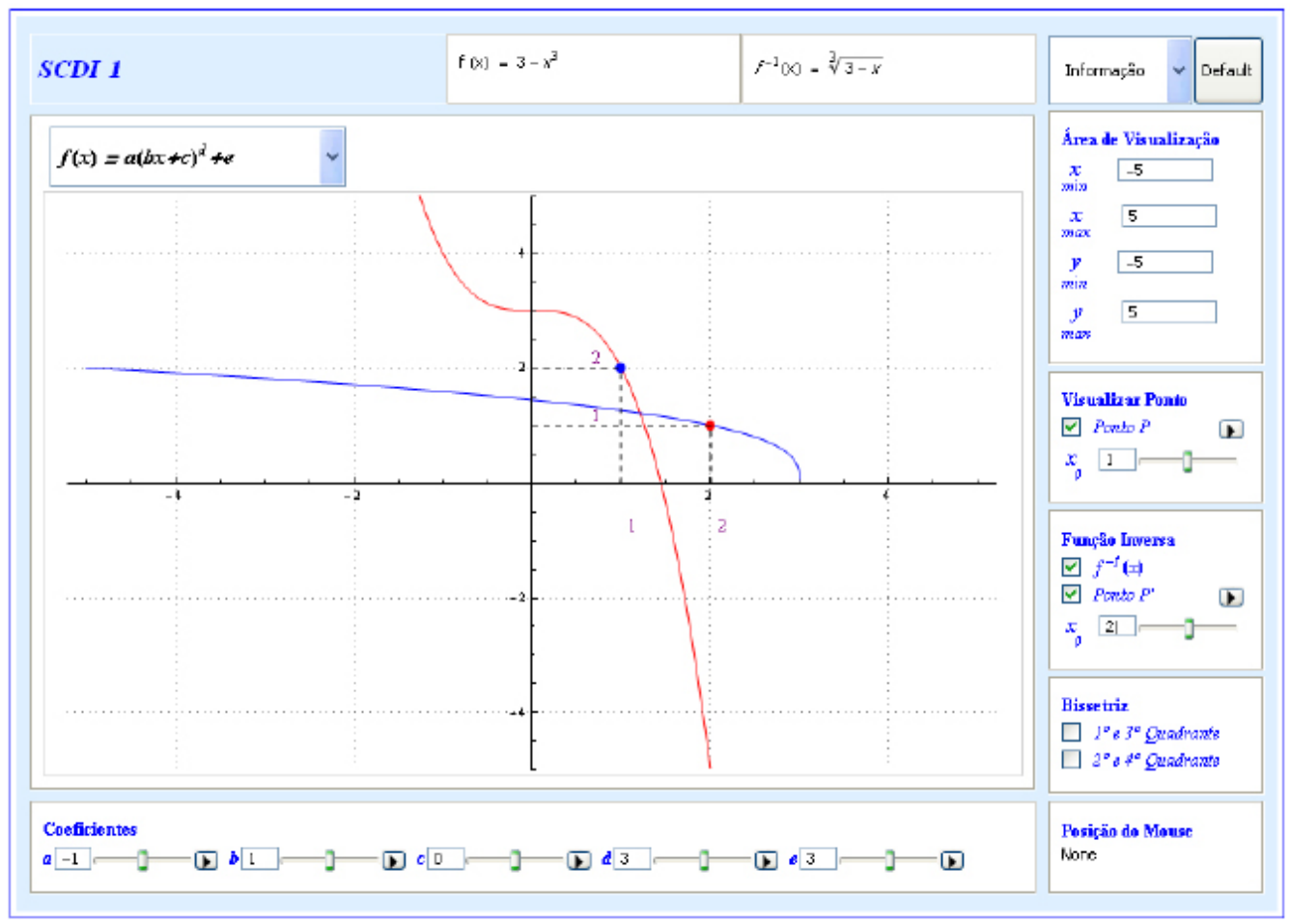

Figura 3. SCDI $1-f(x)=3-x^{3}$ e $f(x)^{-1}=\sqrt[3]{3-x}$

\section{Bissetriz}

Visualização das bissetrizes relativas aos quadrantes pares e ímpares (Figura 4).

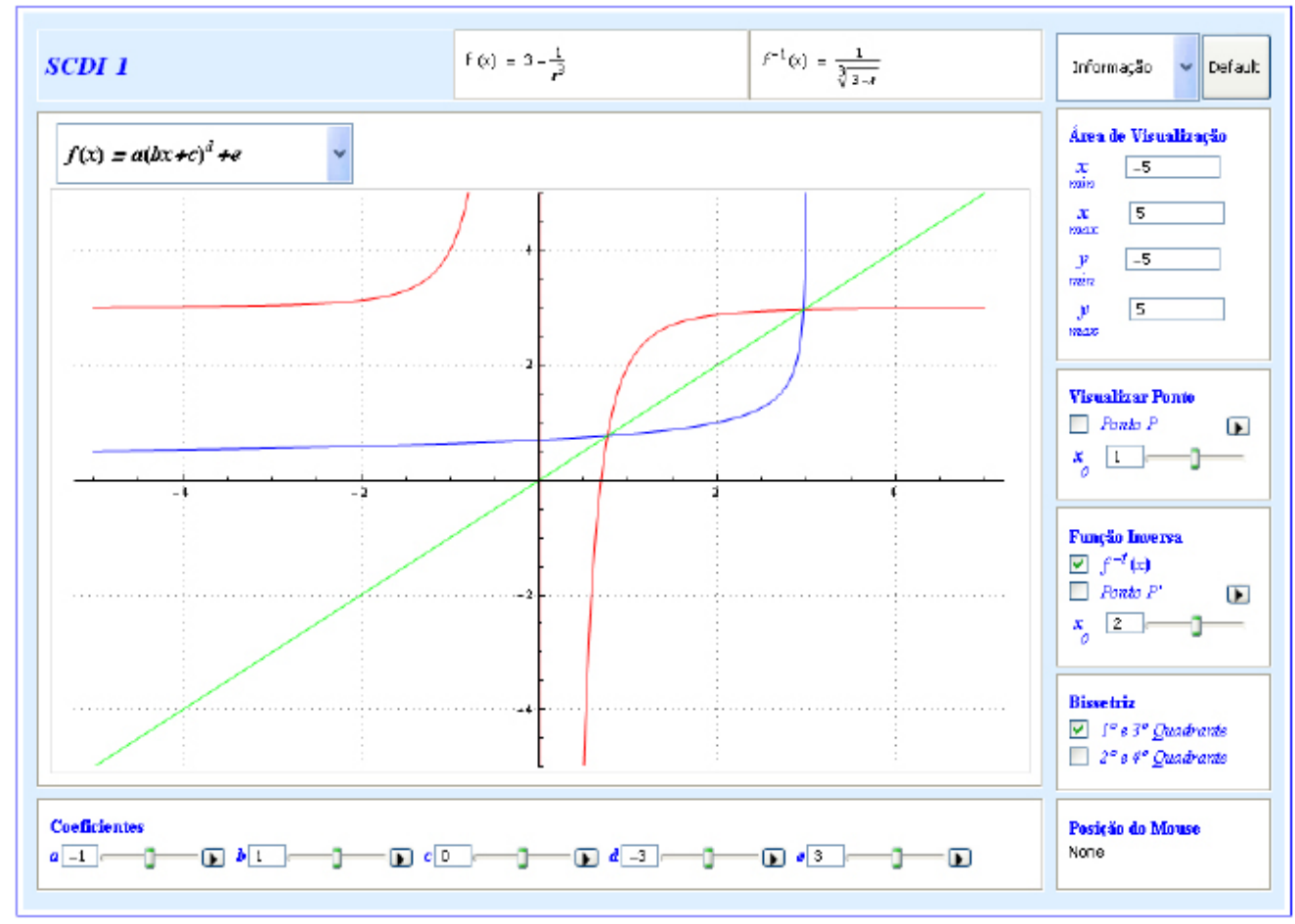

Figura 4. SCDI 1 - Bissetriz ímpar, $f(x)=3-\frac{1}{x^{3}}$ e $f(x)^{-1}=\frac{1}{\sqrt[3]{3-x}}$

Revista Eletrônica TECCEN, Vassouras, v. 4, n. 2, p. 5-14, mai./ago., 2011 


\section{Posição do Mouse}

Determinação das coordenadas do mouse em relação ao plano cartesiano.

\section{Coeficientes}

Modelagem da função através da alteração dos coeficientes $a, b, c, d, e$, adotando como critério de entrada, permitindo também, a interação e animação (slider e player) como visto anteriormente.

\section{Consideração Finais}

Partindo da premissa de que as tecnologias aplicadas ao ensino elevam significativamente o complemento do processo ensino-aprendizagem, o software SCDI 1 tem como objetivo, além de dinamizar as aulas, solidificar conteúdos relativos a disciplina de Cálculo Diferencial e Integral, possibilitando também, o apoio em assuntos voltados ao ensino médio.

Como proposta futura no desenvolvimento do software, objetiva-se na implementação de assuntos relativos à combinação e composição de funções, limites, funções contínuas, derivadas e integrais, concluindo o conteúdo proposto na disciplina de Cálculo I. A próxima etapa desse projeto é a avaliação/validação a partir de sua utilização em sala de aula. 


\section{Referências}

Almeida, M. E. (2000) PROINFO: Informática e formação de professores. Secretaria de Educação a Distância. Brasília: Ministério da Educação. Vol.1, Seed.

Borba, M. C., Penteado, M. G. (2003) Informática e Educação Matemática. 3.ed. Belo Horizonte: Autêntica.

Connally, E. Funções para Modelar Variações - Uma Preparação para o Cálculo. Editora LTC, 3a Edição.

Giraffa , L. M. (1999) Uma arquitetura de tutor utilizando estados mentais. Tese de Doutorado. Porto Alegre: CPGCC/UFRGS.

Myrrha, B. N. ; Carvalho, C. V. A. (2011) INTNUM: Uma Ferramenta Computacional para Visualização e Cálculo dos Métodos de Integração Numérica. Revista Eletrônica TECCEN (ISSN: 1984-0993), Vassouras, v. 3, n. 2, Edição Especial, p. 71-84, abr./ jun.

Papert, S. (1985) A máquina das crianças: repensando a escola na era da informática. Porto Alegre, Artes Médicas, 1994. Logo: computadores e educação. São Paulo, Brasiliense.

Valente, J. A. (2003) Diferentes Usos do Computador na Educação. Em J.A. Valente (Org.), Computadores e Conhecimento: repensando a educação (pp.1-23). Campinas, SP: Gráfica da UNICAMP.

Wolfram, S. (2011) acessado em 01 de agosto de 2011. In: http://www.wolfram.com. 